\title{
Derivation of Theoretical Gravity Model on the Clarke 1880 Ellipsoid for Practical Local Geoid Model Determination
}

\author{
"Eteje S. O., Oduyebo O. F. and Ono M. N. \\ Department of Surveying and Geoinformatics \\ Nnamdi Azikiwe University, Awka, \\ Anambra State, Nigeria \\ *Corresponding Author: eteje.sylvester@yahoo.com \\ DOI: $10.31364 / S C I R J / v 7 . i 2.2019 . P 0219612$ \\ http://dx.doi.org/10.31364/SCIRJ/v7.i2.2019.P0219612
}

\begin{abstract}
The application of gravity anomalies for gravimetric geoid model determination has necessitated the computation of normal gravity on the WGS 84 ellipsoid using the International Gravity Formula. For local gravimetric geoid model determination, the gravity anomalies are computed on the local ellipsoid adopted for geodetic computation in the area/region of study. To determine precise local gravimetric geoid model in countries where Clarke 1880 ellipsoid is adopted for geodetic computation, the theoretical gravity must be computed on the adopted geodetic computation surface. As a result, this paper derives a local theoretical gravity model on the Clarke 1880 ellipsoid for practical local geoid model determination. The model was derived using the Clarke 1880 ellipsoid parameters, angular velocity, gravitational constant and the Somiglinana's closed formula for normal gravity. The derived model was presented in two forms, model A and model B. The two forms of the derived model were compared to determine their suitability as well as agreement. The comparison results show that the two forms of the model agree. Thus, any of the model forms can be applied for accurate normal gravity computation where Clarke 1880 ellipsoid is adopted as a computation surface.
\end{abstract}

Keywords: normal, theoretical, gravity, Clarke 1880, ellipsoid, gravimetric, local geoid, model

\section{INTRODUCTION}

One of the applications of gravity data is geoid modelling. These data are acquired using either satellite observation or gravity meters known as gravimeters. The gravity data, whether acquired by satellite means or gravimeter, they are normally reduced to the geoid. The geoid according to Eteje et al (2018), is the surface which coincides with that surface to which the oceans would conform over the entire earth, if free to adjust to the combined effects of the earth's mass attraction (gravitation) and the centrifugal force of the Earth's rotation. It is a surface adopted as a reference for vertical coordinate system. Reducing the gravity data/observation to the geoid implies that the observation is astronomical. To apply these observations for geoid model determination, their corresponding theoretical gravity of the observed points on a specified ellipsoid is required. The theoretical gravity is a latitude dependent component on the ellipsoid. Thus, it is geodetically as well as geometrically obtained. The difference between the observed/absolute gravity and the theoretical gravity at a point is known as the gravity anomaly.

The gravity anomalies of observed points can be either global or local depending on the ellipsoid on which they were obtained. If the theoretical gravity are computed on the WGS 84 (global) ellipsoid, the theoretical gravity are said to be global. On the other hand, if they are computed on the local ellipsoid adopted for geodetic computation in a country or region, the theoretical gravity are said to be local. The computation of the global gravity anomalies requires the application of the International Gravity Formula. The International Gravity Formula as given by Aziz et al (2010) is

$$
g_{T}=9.7803267714\left(\frac{1+0.00193185138639 \sin ^{2} \phi}{\left(1-0.00669437999013 \sin ^{2} \phi\right)^{1 / 2}}\right) \mathrm{ms}^{-2}
$$

There have been no derived/specified models for computation of normal or theoretical gravity on various local ellipsoids in the world. Precise practical local geoid determination requires the theoretical gravity to be computed on the specified ellipsoid adopted for geodetic computation in the area/region of study. Computing the normal gravity on the local ellipsoid enables the gravity anomalies of observed points to be locally determined. Clarke 1880 is the Nigeria Minna Datum ellipsoid. Several local gravimetric geoid models have been determined in various parts of the country, but the gravity anomalies of selected/observed points were computed using the International Gravity Formula which is global. These were done as a result of the absence of a local theoretical gravity model. Consequently, this paper derives a local theoretical gravity model on the Clarke 1880 ellipsoid for practical local geoid model determination. 
The derivation of the local theoretical gravity model on the Clarke 1880 ellipsoid involves the computation of some parameters such as semi-minor axis, $b$, first eccentricity squared, $e^{2}$, second eccentricity, $e^{\prime 2}$, acceleration rate, $m, q_{o}^{\prime}, q_{o}$, theoretical gravity at the equator, $g_{e}$ and theoretical gravity at the pole, $g_{P}$ using the Clarke 1880 ellipsoid parameter such as such as semimajor axis and flattening, and some other constants such as angular velocity and gravitational constant.

\section{Clarke 1880 Ellipsoid}

The Clarke 1880 ellipsoid is a local ellipsoid adopted for geodetic computation in Nigeria. The semi-major axis $a$ and flattening, $f$ of the Clarke 1880 ellipsoid as given by Eteje et al (2018) are respectively 6378249.145m and 1/293.465.

\section{The Gravity Formula}

The general gravity formula known as the Somiglinana's closed formula for normal gravity was used for the derivation of the International Gravity Formula on the WGS 84 ellipsoid. Moritz (1980) gave the Somiglinana's closed formula for normal gravity as

$$
\gamma=g_{T}=\frac{a g_{e} \cos ^{2} \varphi+b g_{p} \sin ^{2} \varphi}{\sqrt{a^{2} \cos ^{2} \varphi+b^{2} \sin ^{2} \varphi}}
$$

Where, $\gamma=g_{T}=$ point theoretical gravity

$g_{e}=$ theoretical gravity at the equator

$g_{p}=$ theoretical gravity at the pole

$\varphi=$ observed point latitude

$a=$ semi-major axis of specified ellipsoid

$b=$ semi-minor axis of specified ellipsoid

The more convenient form of equation (2) for numerical computation is given by Moritz (1980) as

$$
g_{T}=\frac{g_{e}\left(1+k \sin ^{2} \varphi\right)}{\left(1-e^{2} \sin ^{2} \varphi\right)^{1 / 2}}
$$

with $k$ given by Madden (2006) as

$$
k=\frac{b g_{p}}{a g_{e}}-1
$$

Where, $e^{2}$ is the eccentricity squared of the specified ellipsoid.

The computation of $k$ requires the computation of the theoretical gravity at the equator and the pole. The theoretical gravity at the equator and the pole are respectively computed as (Moritz, 1980)

$$
\begin{aligned}
& g_{e}=\frac{G M}{a b}\left(1-m-\frac{m}{6} \cdot \frac{e^{\prime} q_{o}^{\prime}}{q_{o}}\right) \\
& g_{p}=\frac{G M}{a^{2}}\left(1+\frac{m}{3} \cdot \frac{e^{\prime} q_{o}^{\prime}}{q_{o}}\right)
\end{aligned}
$$

Where,

$$
q_{o}^{\prime}=3\left(1+\frac{1}{e^{\prime 2}}\right) \cdot\left(1-\frac{1}{e^{\prime}} \arctan e^{\prime}\right)-1
$$




$$
\begin{aligned}
& 2 q_{o}=\left(1+\frac{3}{e^{\prime 2}}\right) \cdot \arctan e^{\prime}-\frac{3}{e^{\prime}} \\
& m=\text { acceleration rate }=\frac{\omega^{2} a^{2} b}{G M} \\
& G M=\text { gravitational constant }=3986005 \times 10^{8} \mathrm{~m}^{3} \mathrm{~s}^{-2} \\
& \omega^{2}=\text { angular velocity }=7292115 \times 10^{-11} \mathrm{rad} \mathrm{s}^{-1}
\end{aligned}
$$

Also, the computation of the acceleration rate, $m, q_{o}^{\prime}$ and $q_{o}$, necessitates the computation of the semi-minor axis, first eccentricity squared and second eccentricity squared of the specified ellipsoid. The semi-minor axis of an ellipsoid is computed as (Clynch, 2002)

$$
\begin{aligned}
& f=\frac{a-b}{a} \\
& \Rightarrow b=a(1-f)
\end{aligned}
$$

while the first eccentricity squared is given as (Li and Gotze, 2001)

$$
e^{2}=\frac{a^{2}-b^{2}}{a^{2}}
$$

The second eccentricity squared according to Moritz (1980) is

$$
e^{\prime 2}=\frac{a^{2}-b^{2}}{b^{2}}=\frac{e^{2}}{\left(1-e^{2}\right)}
$$

Where,

$$
\begin{aligned}
& f=\text { flattening of the ellipsoid } \\
& e=\text { first eccentricity } \\
& e^{\prime 2}=\text { second eccentricity squared }
\end{aligned}
$$

The conventional abbreviated series expansion for the gravity formula is (Moritz, 1980):

$$
\gamma=g_{T}=g_{e}\left(1+f^{*} \sin ^{2} \varphi-\frac{1}{4} f_{4} \sin ^{2} 2 \varphi\right)
$$

Where,

$$
\begin{aligned}
& f^{*}=\text { gravity flattening }=\frac{g_{p}-g_{e}}{g_{e}} \\
& f_{4}=\frac{1}{2} f^{2}+\frac{5}{2} f m
\end{aligned}
$$

\section{Gravity Anomaly}

The gravity anomaly is the difference between the points gravity reduced to the geoid and the latitude as well as the normal gravity computed on a specified ellipsoid and corrected for free air and effect of rock. The points gravity anomalies are grouped into two: the free air gravity anomalies and the Bouguer gravity anomalies. The free air gravity anomaly, $\Delta g_{f a}$ is computed as (Aziz et al, 2010):

$$
\Delta g_{f a}=g_{o b s}-g_{f a}-g_{o}
$$

Where, $\Delta g_{f a}$ is free-air anomaly, $g_{o b s}$ is observed gravity and $g_{o}$ is theoretical gravity and $g_{f a}=0.3086[\mathrm{mGa} 1 / \mathrm{m}] \mathrm{H}$. 
The Bouguer gravity anomaly, $\Delta g_{B}$ is given by Vermeer (2016) as

$$
\Delta g_{B}=\Delta g_{f a}-0.1119 H
$$

The orthometric heights of the points can be obtained from the Digital Terrain Model of the area.

\section{Gravimetric Geoid}

The gravimetric method of geoid determination can be carried out by the well-known Stokes-integral, equation (19) and the use of accurately determined absolute gravity data (Heiskanen and Moritz, 1967, Eteje, 2015 and Eteje et al, 2018).

$$
N=\frac{R}{4 \pi \gamma} \iint_{\sigma} \Delta g S(\psi) d \sigma
$$

Where, $N$ is geoid undulation, $\Delta g$ is gravity anomaly, $S(\psi)$ is stokes function, $\gamma$ is normal gravity on the reference ellipsoid and $R$ is mean radius of the earth.

\section{DERIVATION OF THE CLARKE 1880 THEORETICAL GRAVITY MODEL}

The derivation of the Clarke 1880 theoretical gravity model involves the computation of the following: semi-minor axis, $b$, first eccentricity squared, $e^{2}$, second eccentricity squared, $e^{\prime 2}$, acceleration rate, $m, q_{o}^{\prime}, q_{o}$, theoretical gravity at the equator, $g_{e}$, theoretical gravity at the pole, $g_{P}, k$, and the presentation of the model in its final forms. Figure 1 shows the flow chart of the computation procedures.

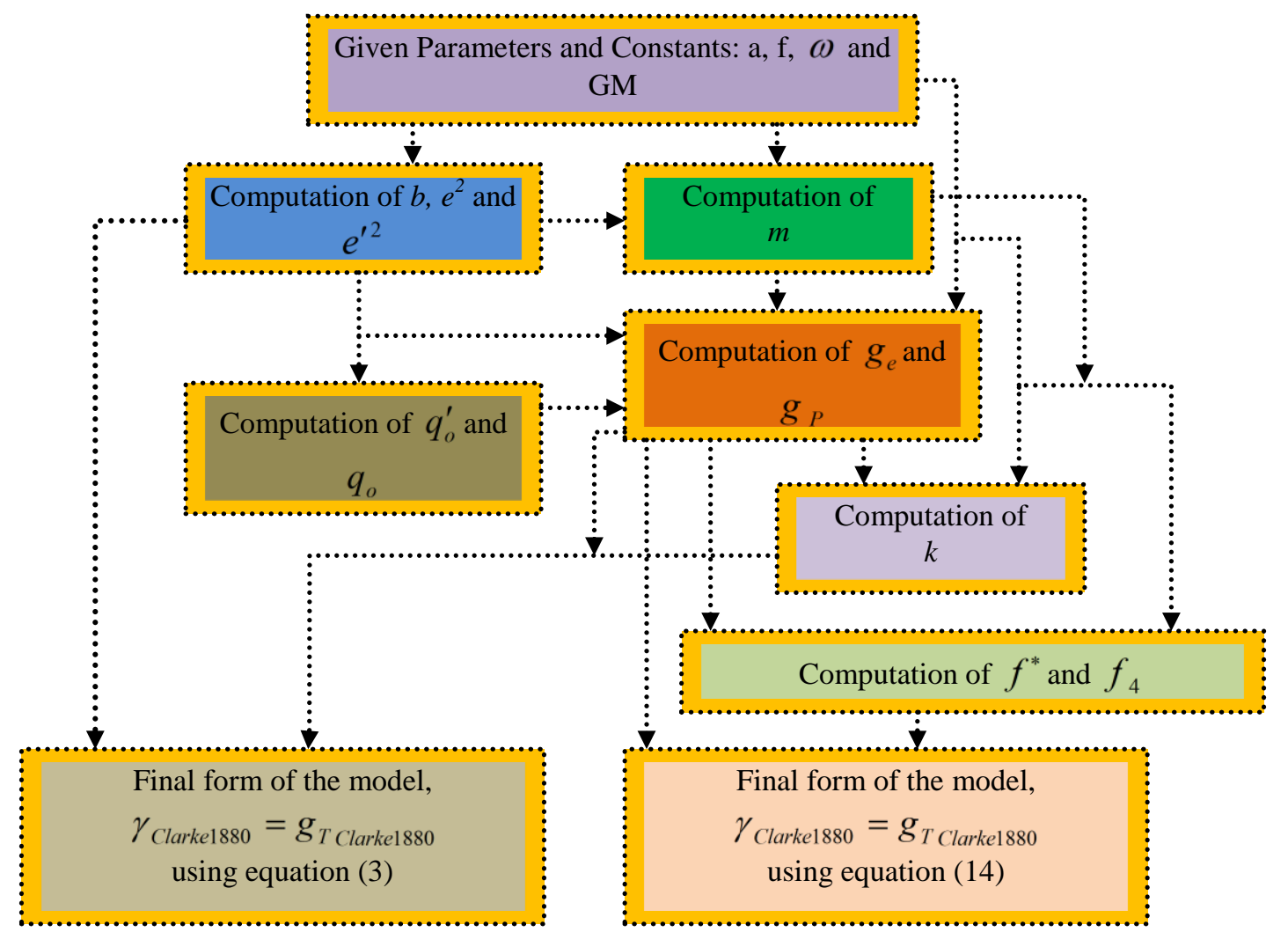

Fig. 1: Flow Chart of the Computation Procedures

Using the given semi-major axis and flattening of the Clarke 1880 ellipsoid, the angular velocity and gravitational constant, the computations were carried out as follow:

\section{Computation of Semi-minor Axis, $b$}


The semi-minor axis was computed using equation (11) as

$$
b=6378249.145\left(1-\frac{1}{293.465}\right)=6356514.869 \mathrm{~m}
$$

\section{Computation of First Eccentricity Squared, $e^{2}$}

Using equation (12), the first eccentricity squared was computed as

$$
e^{2}=\frac{6378249.145^{2}-6356514.869^{2}}{6378249.145^{2}}=0.00680351145465242 \mathrm{~m}
$$

\section{Computation of Second Eccentricity Squared, $e^{\prime 2}$}

The second eccentricity squared was computed with equation (13) as

$$
e^{\prime 2}=\frac{6378249.145^{2}-6356514.869^{2}}{6356514.869^{2}}=0.006850115 \mathrm{~m}
$$

\section{Computation of Acceleration Rate, $m$}

The acceleration rate, $m$ was computed using equation (9) as

$$
m=\frac{\left(7292115 \times 10^{-11}\right)^{2} \times 6378249.145^{2} \times 6356514.869}{3986005 \times 10^{8}}=0.003449778
$$

\section{Computation of $q_{o}^{\prime}$}

With equation (7), $q_{o}^{\prime}$ was computed as

$$
\begin{aligned}
q_{o}^{\prime} & =3 \times\left(1+\frac{1}{0.006850115}\right) \times\left(1-\frac{1}{0.082765427} \times \arctan 0.082765427\right)-1 \\
& =0.002732032
\end{aligned}
$$

\section{Computation of $q_{o}$}

Using equation (8), $q_{o}$ was computed as

$$
\begin{aligned}
2 q_{o} & =\left(1+\frac{3}{0.006850115}\right) \times \arctan 0.082765427-\frac{3}{0.082765427}=0.000150304 \\
\therefore q_{o} & =\frac{0.000150304}{2} \\
& =0.000075152
\end{aligned}
$$

It is to be noted here that the value of arctan 0.082765427 was converted to radian before it was used.

\section{Computation of Theoretical Gravity at the Equator, $g_{e}$}

The theoretical gravity at the equator on the Clarke 1880 ellipsoid, $g_{e}$ was computed using equation (5) as 


$$
\begin{aligned}
g_{e} & =\frac{3986005 \times 10^{8}}{6378249.145 \times 6356514.869}\left(-\frac{0.003449778}{6} \times \frac{0.082765427 \times 0.002732032}{0.000075152}\right) \\
& =9.780519381 \mathrm{~ms}^{-2}
\end{aligned}
$$

\section{Computation of Theoretical Gravity at the Pole, $g_{P}$}

With equation (6), the theoretical gravity at the pole, $g_{p}$ was computed as

$$
\begin{aligned}
g_{p} & =\frac{3986005 \times 10^{8}}{6378249.145^{2}}\left(1+\frac{0.003449778}{3} \times \frac{0.082765427 \times 0.002732032}{0.000075152}\right) \\
& =9.831842301 \mathrm{~ms}^{-2}
\end{aligned}
$$

\section{Computation of $k$}

By substituting the obtained values of $g_{e}$ and $g_{P}$ into equation (4), $k$ was computed as

$$
k=\frac{6356514.869 \times 9.831842301}{6378249.145 \times 9.780519381}-1=0.00182202113732433
$$

Final Forms of the Clarke 1880 Ellipsoid Theoretical Gravity Model, $\gamma_{\text {Clarke } 1880}=g_{\text {TClarke } 1880}$

Also, by substituting the values of $g_{e}, k$, and $e^{2}$ into equation (3), the final form of the Clarke 1880 ellipsoid theoretical gravity model A was obtained as

$$
\gamma_{\text {Clarke } 1880(A)}=g_{\text {TClarke } 1880(A)}=9.780519381\left[\frac{\left(1+0.00182202113732433 \sin ^{2} \phi\right)}{\left(1-0.00680351145465242 \sin ^{2} \phi\right)^{1 / 2}}\right] \mathrm{ms}^{-2}
$$

Again, using the series expansion formula given in equations (14) to (16), the series expansion form of the model (model B) was obtained as follow:

$f^{*}$ was computed using equation (15) as

$$
f^{*}=\frac{9.831842301-9.780519381}{9.780519381}=0.00524746365716546
$$

while $f_{4}$ was computed with equation (16) as

$$
\begin{aligned}
f_{4} & =\frac{1}{2} \times 0.00340756137869933^{2}+\frac{5}{2} 0.00340756137869933 \times 0.003449778 \\
& =0.00003519406296951
\end{aligned}
$$

Substituting the computed values of $f^{*}$ and $f_{4}$ into equation (14), we obtained

$$
\begin{aligned}
\gamma=g_{T} & =9.780519381\left(1+0.00524746365716546 \sin ^{2} \varphi-\frac{1}{4} \times 0.00003519406296951 \sin ^{2} 2 \varphi\right) \mathrm{ms}^{-2} \\
& =9.780519381\left(1+0.00524746365716546 \sin ^{2} \varphi-0.0000087985157424 \sin ^{2} 2 \varphi\right) \mathrm{ms}^{-2}
\end{aligned}
$$

Therefore, the series expansion form (model B) of the Clarke 1880 ellipsoid theoretical gravity model is 


$$
\gamma_{\text {Clarke } 1880(B)}=g_{\text {TClarke } 1880(B)}=9.780519381\left(1+0.0052474636 \sin ^{2} \varphi-0.0000087985 \sin ^{2} 2 \varphi\right) \mathrm{ms}^{-2}
$$

\section{Comparison of the Models}

Table 1 and figures 2 and 3 show the comparison of the two model forms. The two model forms, that is, Model A obtained with equation (3) and model B obtained with equation (14) as well as using the series expansion formula were compared to determine the suitability and agreement of the model forms. The theoretical gravity of six points were computed with their latitudes using model A and model B. The computed normal gravity of the points using the two model forms were compared to obtain their differences. It can be seen from table 1 that the normal gravity of the six points obtained from model A are identical with those obtained from modal B which implies that there is no difference between the computation results of the two model forms. Thus, any of the model forms can be applied where Clarke 1880 ellipsoid is adopted as a reference surface for geodetic computation. It can also be seen from figures 2 and 3 that the plotted normal gravity from the two model forms have the same shape as well as their differences which also implies the agreement and suitability of the two model forms.

Table 1: Comparison of the Models

\begin{tabular}{|c|c|c|c|}
\hline Latitude & Model A (Equation (3)) & Model B (Equation (14)) & Difference \\
\hline 6.331514607 & 9.781140782 & 9.781139430 & 0.000001352 \\
\hline 6.301664360 & 9.781134960 & 9.781133620 & 0.000001339 \\
\hline 6.287544686 & 9.781132215 & 9.781130881 & 0.000001334 \\
\hline 6.257952119 & 9.781126483 & 9.781125161 & 0.000001321 \\
\hline 6.237847990 & 9.781122603 & 9.781121290 & 0.000001313 \\
\hline 6.214210013 & 9.781118058 & 9.781116755 & 0.000001303 \\
\hline
\end{tabular}

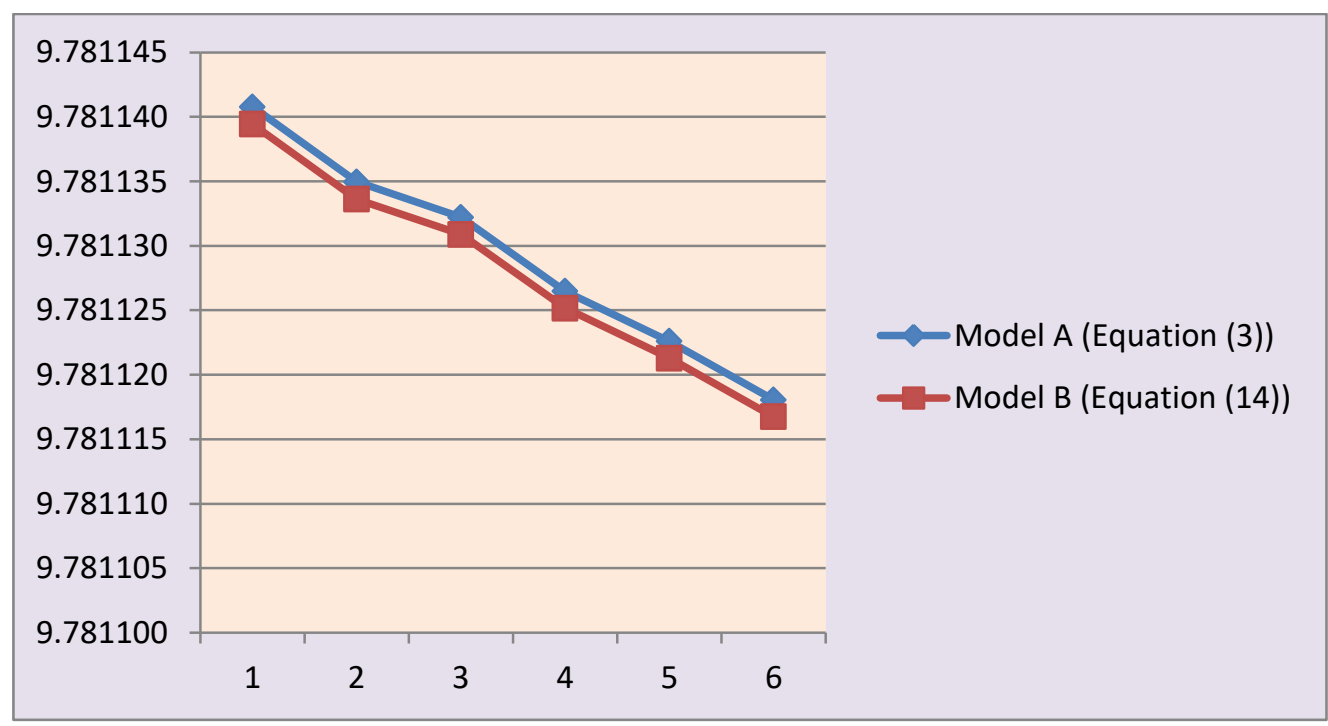

Fig. 2: Plot of the Computed Normal gravity 


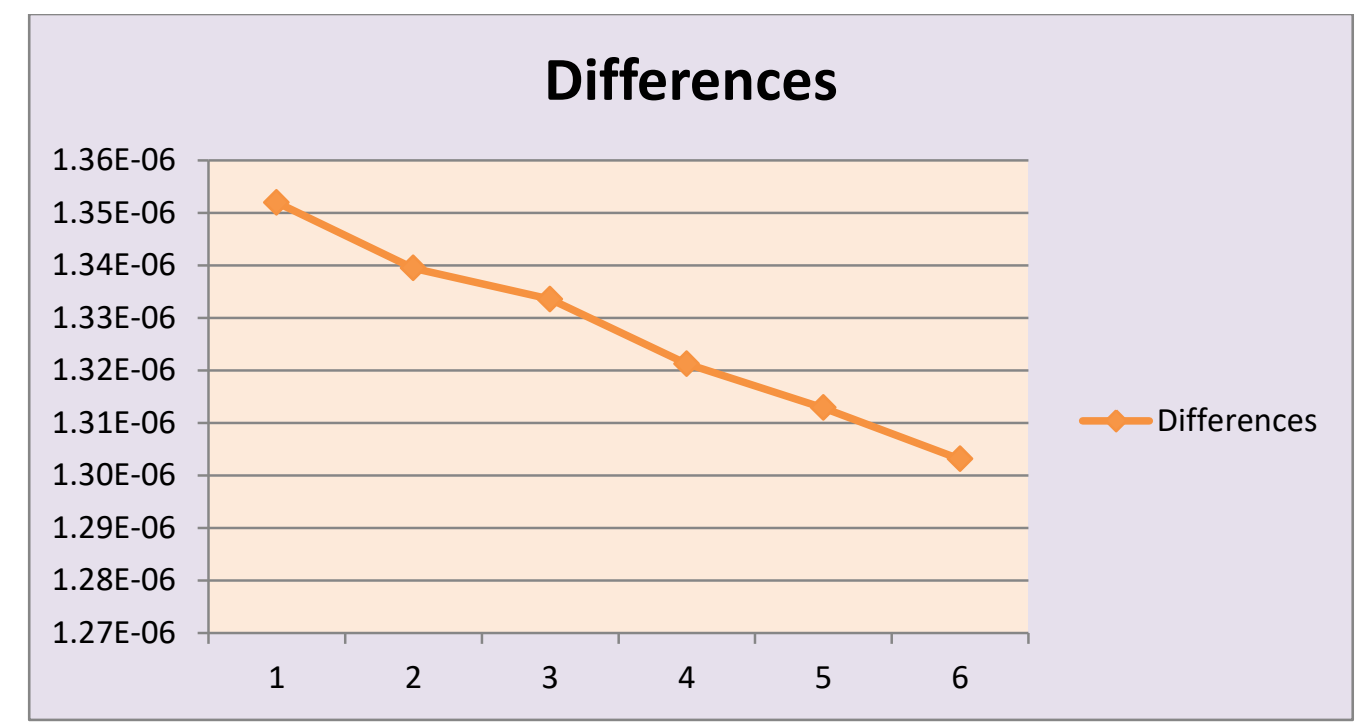

Fig. 3: Plot of the Differences of the Computed Normal gravity

\section{CONCLUSION}

Seeing that the gravimetric method of geoid model determination requires the use of gravity anomalies of selected points, the normal gravity of points of known absolute or observed gravity are required to be computed. For precise practical local geoid model determination, the normal gravity of the selected points are computed on the local ellipsoid adopted for geodetic computation in the region/area of study. Since Clarke 1880 is a local ellipsoid adopted for geodetic computation in some countries such as Nigeria, this paper has derived a local theoretical gravity model on the Clarke 1880 ellipsoid for practical local geoid model determination. The derived model was presented in two forms: model A and model B. The two model forms were compared to determine their suitability as well as agreement. The comparison results show that the two forms of the derived model agree. Thus, any of the model forms can be applied for accurate normal gravity computation in regions/countries where Clarke 1880 ellipsoid is adopted as a reference surface for geodetic computation.

\section{REFERENCES}

Aziz, N., Majid, B. and Jörg, E. (2010): Gravity and Magnetic Data Acquisition Over a Segment of the Møre-Trøndelag Fault Complex. NGU Report 2010.049.

Clynch, J. R. (2002): Notes on Gravity. Naval Postgraduate School. https://docplayer.net/26505279-Notes-on-gravity-james-rclynch-naval-postgraduate-school-2002.html.

Eteje S. O., Oduyebo O. F. and Olulade S. A. (2018): Comparative Analysis of Three Geodetic Datum Transformation Software for Application between WGS84 and Minna Datums. International Journal of Engineering Science and Computing, Vol. 8, No.12, pp 19410- 19417.

Eteje S. O., Oduyebo O. F. and Olulade S. A. (2018): Procedure for the Determination of Local Gravimetric-Geometric Geoid Model. International Journal of Advances in Scientific Research and Engineering (IJASRE), Vol. 4, No 8, pp $206-214$. http://doi.org/10.31695/IJASRE.2018.32858.

Eteje, S. O. (2015): Determination of the Local Geoid Model Using the Geometric (GPS/Levelling) Method for Evboriaria in Benin City, Edo State. Unpublished MSc Thesis of the Department of Surveying and Geoinformatics, Nnamdi Azikiwe University, Awka.

Eteje, S. O., Ono, M. N. and Oduyebo, F. O. (2018): Practical Local Geoid Model Determination for Mean Sea Level Heights of Surveys and Stable Building Projects. IOSR Journal of Environmental Science, Toxicology and Food Technology (IOSRJESTFT), Vol. 12, No 6, PP 30-37.

Heiskanen, W. A. and Moritz, H. (1967): Physical Geodesy. W. H. Freeman and Company, San Francisco in Eteje, S. O. (2015): Determination of the Local Geoid Model Using the Geometric (GPS/Levelling) Method for Evboriaria in Benin City, Edo State. Unpublished M.Sc Thesis of the Department of Surveying and Geoinformatics, Nnamdi Azikiwe University, Awka.

Li, X. and Gotze, H. (2001): Tutorial: Ellipsoid, Geoid, Gravity, Geodesy, and Geophysics. Geophysics, Vol. 66, No. 6 p p 16601668.

Madden, M. M. (2006): Gravity Modeling for Variable Fidelity Environments. NASA, Hampton,VA,23681.https://ntrs.nasa.gov/archive/nasa/casi.ntrs.nasa.gov/2006002864.pdf. Accessed February 14, 2019.

Moritz, H. (1980): Geodetic Reference System 1980. http://geoweb.mit.edu/ tah/12.221_2005/grs80_corr.pdf. Accessed February 14,

Vermeer, M. (2016): Physical Geodesy. https://users.aalto.fi/ mvermeer/mpk-en.pdf. Accessed February 2, 2018. 